\title{
Fabrication of reduced graphene oxide modified poly(3,4-ethylenedioxythiophene) polystyrene sulfonate based transparent conducting electrodes for flexible optoelectronic application
}

\author{
Sandeep Sharma ${ }^{1} \cdot$ Karamvir Singh $^{1} \cdot$ Sandeep Kumar ${ }^{1} \cdot$ Kapil Bhatt $^{1} \cdot$ Yashashchandra Dwivedi $^{2}$. \\ Abhimanyu Rana ${ }^{3} \cdot$ C. C. Tripathi ${ }^{1}$
}

Received: 2 June 2020 / Accepted: 21 December 2020 / Published online: 7 January 2021

(c) The Author(s) 2020 OPEN

\begin{abstract}
Present article reports on reduced graphene oxide ( $\mathrm{rGO}$ ) modified poly(3,4-ethylenedioxythiophene) polystyrene sulfonate (PEDOT: PSS) based transparent conducting electrodes for flexible optoelectronic applications. PEDOT: PSS samples embedded with different $\mathrm{rGO}$ concentrations i.e. 0, 1, 2, 3, 4, 5 wt\% were prepared and later on, bar coated on polyethylene terephthalate substrate using a $30 \mu \mathrm{m}$ wire size bar. Various parameters including sheet resistance, bending test (outside and inside bending), optical transmittance etc. were estimated. Our analysis indicates that the samples with 1 wt\% rGO possess improved results i.e. low sheet resistance $(315 \pm 8 \Omega / \mathrm{sq}$.) and high transmittance ( $74 \%)$. Additionally, the sample shows low electrical resistance variation up to $12 \%$ (maximum increase) during outward bending and $9 \%$ (maximum decrease) during inward bending of the sample for bending curvature from 20 to $100 \mathrm{~m}^{-1}$.
\end{abstract}

Keywords Transparent conducting electrode $\cdot$ Poly (3 - 4-ethylenedioxythiophene): poly (styrene sulfonic acid) . Dimethyl sulfoxide $\cdot$ Optoelectronics devices · Bar coating

\section{Introduction}

Transparent conducting electrode (TCE) is an essential part of various optoelectronic devices such as lightemitting diodes, photovoltaic cells, touch screens, solar cells, and flat-panel displays [1-3]. Among various TCEs, Indium tin oxide (ITO) is most preferred as it wealth with attracting characteristics like high transmittance ( 90\%) and low sheet resistance ( $\sim 10 \Omega /$ sq.) [4]. Still, the quest to develop improved materials for flexible TCE applications is on, as ITO possesses practical limitations of its brittle nature. Additionally, limited indium resources restrict the prolonged run use for future flexible optoelectronics devices. Hence, there is a requirement of alternate material for ITO as TCE. Several TCE materials like transparent conducting polymers (TCPs), metal nanowires, carbon nanotubes, and graphene have been explored for replacing ITO $[4,5]$. Among these materials, both graphene and TCPs play a vital role due to their lightweight, low sheet resistance, high transparency, and flexibility [6-8]. Chemical vapor deposition (CVD) is the most preferred technique to deposit graphene. However, the CVD technique requires high temperatures $\left(\sim 1000{ }^{\circ} \mathrm{C}\right)$ operation under a high vacuum, making it infeasible to deposit graphene directly on flexible (polymer) substrates [9]. Some of the transfer processes have been explored to deposit graphene on

$\checkmark$ Sandeep Sharma, er.sandeep46@gmail.com | ${ }^{1}$ University Institute of Engineering and Technology, Kurukshetra University, Kurukshetra 136119, India. ${ }^{2}$ Physics Department, National Institute of Technology Kurukshetra, Kurukshetra 136119, India. ${ }^{3}$ Physics Department, Centre for Advanced Materials \& Devices, BML Munjal University, Gurugram 122413, India. 
polymer substrates using CVD [10], however challenges remain persists in discovering suitable mechanisms for graphene films transfer on flexible substrates without developing cracks during the transfer process, obtaining large-area graphene film with a clean surface and achieving a low-cost transfer process. To undertake CVD technique issues, several solutions have been proposed for the graphene deposition $[11,12]$. Nevertheless, yet no process has been precise enough to deposit graphene on a flexible substrate. Among TCPs, Polyaniline (PANI), Polypyrrole (PPy), and PEDOT: PSS are well-known polymers [13-16], but PEDOT: PSS is most preferred materials because of its low sheet resistance and high transmittance $[14,16]$. The conductivity of PEDOT: PSS can be enhanced by chemical treatment with some solvent including sulfuric acid, formic acid, Polyethylene Glycol, N,N-dimethyl formamide, xylitol, tetrahydrofuran, methoxyethanol, and dimethyl sulfoxide (DMSO) [17-26], [27]. Despite this, PEDOT:PSS has several problems such as inhomogeneous electrical properties and poor long-term stability $[28,29]$.

In the present work, for achieving a flexible TCE as an alternate of ITO, rGO modified PEDOT: PSS TCE was fabricated on a flexible PET sheet using a bar coating technique with high performance and stability. At first, graphene oxide (GO) was synthesized using modified Hummer's method and then rGO was synthesized by reducing the GO using L-ascorbic acid. The different proportion of synthesized $\mathrm{rGO}$ was mixed in PEDOT: PSS solution and deposited on a PET sheet using bar coating technique as explained in Sect. 2. The deposited film was further exposed to the DMSO solution. Vibrational analysis including Raman spectra, FTIR absorption measurement; sheet resistance, flexibility, of the fabricated electrodes was estimated. The results and conclusion of the work is discussed in Sect. 3, 4 respectively.

\section{Materials and methods}

Materials: graphite powder, PEDOT: PSS (1.3 wt\% dispersion in water) were obtained from Sigma Aldrich (USA); dimethyl sulfoxide (DMSO), and polyethylene terephthalate (PET) film were used for making transparent conducting films. In addition to this, soap solution, isopropyl alcohol (IPA), deionized (DI) water, and nitrogen gas were used to prepare the PET substrate for film deposition.

At first, PET substrates $(2.5 \mathrm{~cm} \times 2.5 \mathrm{~cm})$ were cut and cleaned by sonication in soap solution then rinsed in $\mathrm{DI}$ water, after that the substrate was sonicated in isopropyl alcohol, and then rinsed in DI water. The substrates were dried with nitrogen and treated with oxygen plasma for $5 \mathrm{~min}$ each to enhance the wettability and adhesive strength of the substrate. Graphene oxide (GO) was synthesised using a modified Hummer's method. In brief, $3 \mathrm{~g}$ graphite was dissolved in $60 \mathrm{ml}$ of concentrated $\mathrm{H}_{2} \mathrm{SO}_{4}$ using stirring at room temperature for $24 \mathrm{~h}$. Thereafter, the solution was placed in an ice bath to maintain the solution temperature up to $10^{\circ} \mathrm{C}$. Then $3 \mathrm{~g}$ of $\mathrm{NaNO}_{3}$ was added to the solution and stirred for $30 \mathrm{~min}$ by sustaining the temperature $10^{\circ} \mathrm{C}$ in an ice bath, and took out the solution from the ice bath, and stirred it again for $1 \mathrm{~h}$ at room temperature. After that, the solution was diluted with $240 \mathrm{ml}$ of $\mathrm{DI}$ and stirred it for $30 \mathrm{~min}$ and again diluted the solution with $1 \mathrm{~L}$ of DI, after that $45 \mathrm{ml}$ of $\mathrm{H}_{2} \mathrm{O}_{2}$ slowly added drop by drop for $30 \mathrm{~min}$. The resulted solution was washed with $\mathrm{HCl}(5 \% \mathrm{wt}): 65.2 \mathrm{ml}$ of $\mathrm{HCl}$ in $84.8 \mathrm{ml}$ of Dl and filtered with a paper filter and washed to achieve neutral $\mathrm{pH}$. The resulted product was dried at $80^{\circ} \mathrm{C}$ in the oven for $48 \mathrm{~h}$ to receive $\mathrm{GO}$ powder. Thereafter, reduced graphene oxide ( $\mathrm{rGO}$ ) was synthesised by reducing the GO using $\mathrm{L}$-ascorbic acid. Aqueous suspension of $\mathrm{GO}$ was prepared by ultra-sonicating $500 \mathrm{mg}$ of GO in $1 \mathrm{~L} \mathrm{DI}$ for $2 \mathrm{~h}$. Thereafter, $500 \mathrm{mg}$ of ascorbic acid was added and the solution was sonicated for the next $24 \mathrm{~h}$. The resulting suspension was filtered and washed with DI water multiple times and dried at $60^{\circ} \mathrm{C}$ in a vacuum oven.

Different concentrations $0 \%, 1 \%, 2 \%, 3 \%, 4 \%$, and $5 \%$ (wt\%) of rGO was mixed in PEDOT: PSS and sonicated for $4 \mathrm{~h}$ each. The prepared solution was deposited on a PET sheet using a bar coating and treated with DMSO. DMSO is a high boiling solvent and strongly enhanced the conductivity of PEDOT:PSS layer [30]. Respective change in sheet resistance and transmittance of the deposited layers were observed. A schematic diagram of the experiment is given in Fig. 1.

\subsection{Characterization techniques}

The deposited films of rGO modified PEDOT: PSS on PET substrates were characterized by different methods. The sheet resistance of the deposited samples was estimated by the four-probe (Vijayanta Electronics, India) technique. The analysis of surface roughness of the layer was carried out by Atomic Force Microscopy (AFM) using a machine of model Pro 47, provided by NT-MDT spectrum instruments Russia. The optical transmittance measurements were carried out on UV-Vis Spectrophotometer (Rescholar, India). The optical images were taken by optical microscope (Olympus BX53M) and the molecular vibrations of synthesised $\mathrm{GO}$ and $\mathrm{rGO}$ were characterised by Raman spectroscopy via Raman Spectrometer setup (WI Tec Alpha300 RAS) and FTIR absorption via FTIR spectrometer (PerkinElmer Spectrum Two) available in BML Munjal University, Gurugram. The bending test of the deposited samples was performed by taking the relative variation of electrical resistance using Keithley 2450 as a function of 

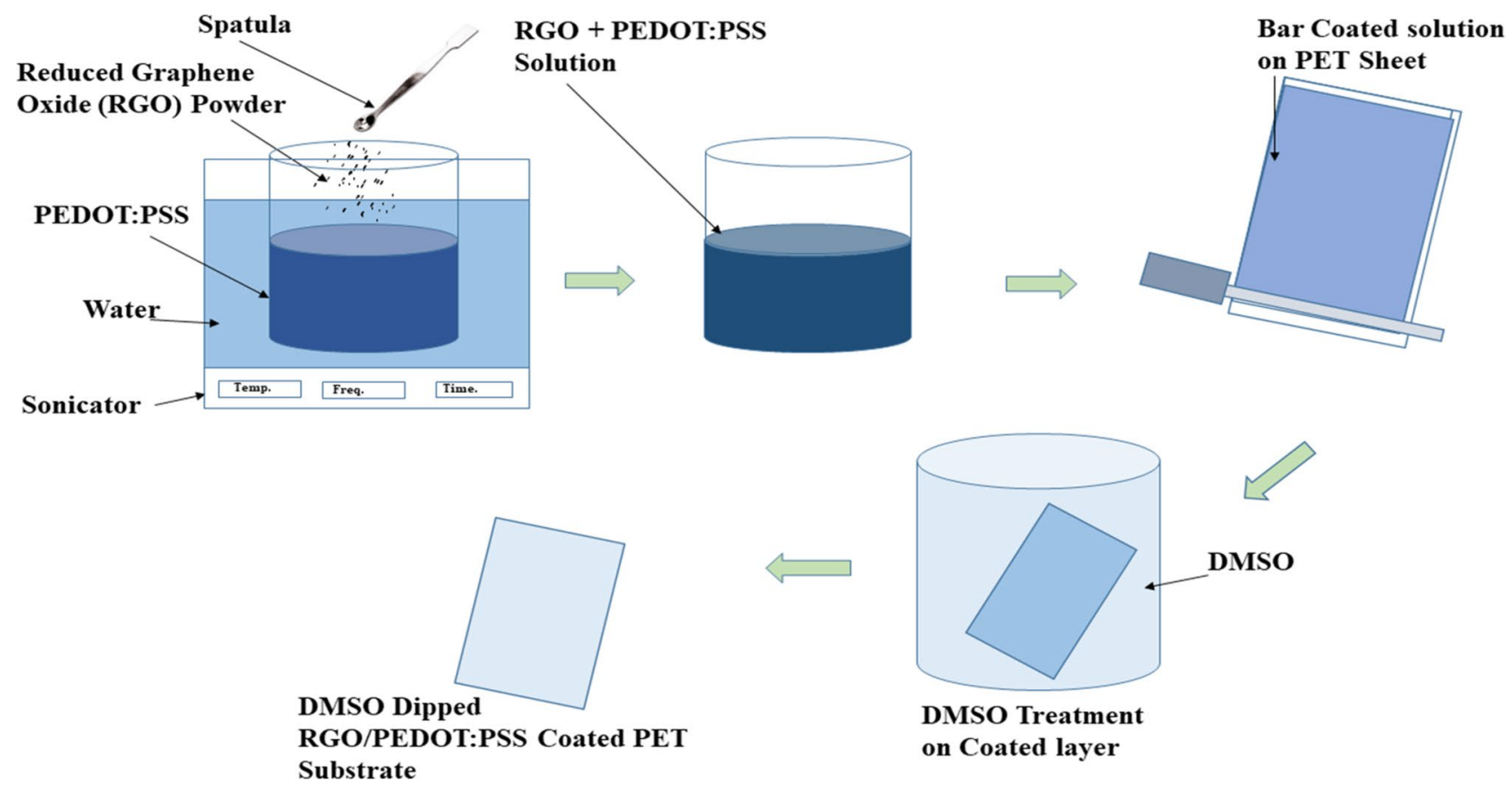

DMSO Treatment

on Coated layer

Fig. 1 Schematic diagram of rGO + PEDOT: PSS solution bar coating processes on PET sheets

bending curvature for both sides including outer bending and inner bending.

\section{Results and discussion}

In this work, rGO/PEDOT: PSS based transparent conducting electrodes were prepared. In rGO synthesis process, first of all GO was synthesised using modified Hummer's method and then it was reduced in $\mathrm{rGO}$ form using $\mathrm{L}$-ascorbic acid. The resulted GO and $\mathrm{rGO}$ were characterised and analysed by Raman and FTIR spectroscopy shown in Fig. 2. Raman spectra of GO and rGO present a D-band at $1310 \mathrm{~cm}^{-1}$ and a G-band at $1609 \mathrm{~cm}^{-1}$. The D peak shows the presence of defects and amorphous structure and the $G$ peak shows the ordered $\mathrm{sp}^{2}$ bond as reported by Pimenta et al. [31]. Due to the double resonance, D' peak at $1730 \mathrm{~cm}^{-1}$ is presents in Raman spectra of both $\mathrm{GO}$ and

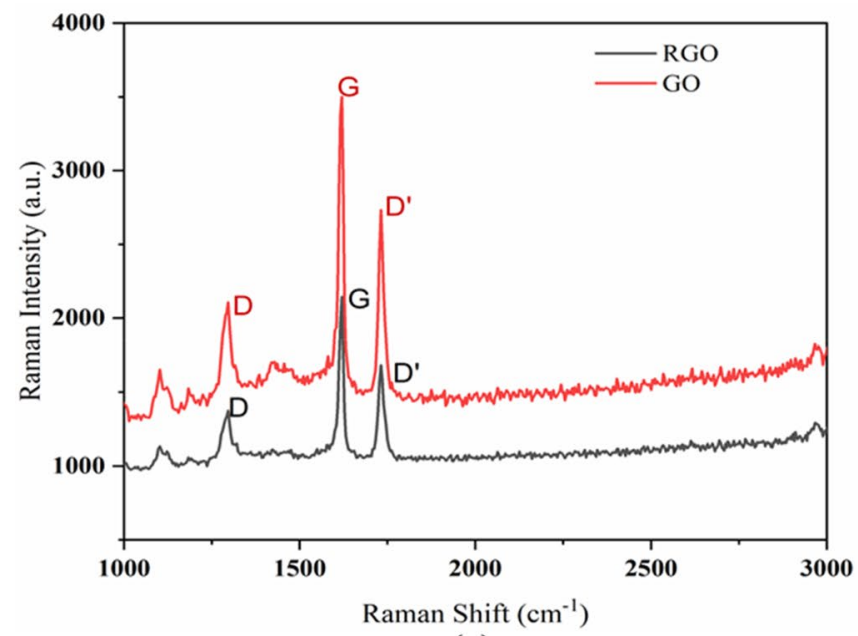

(a)

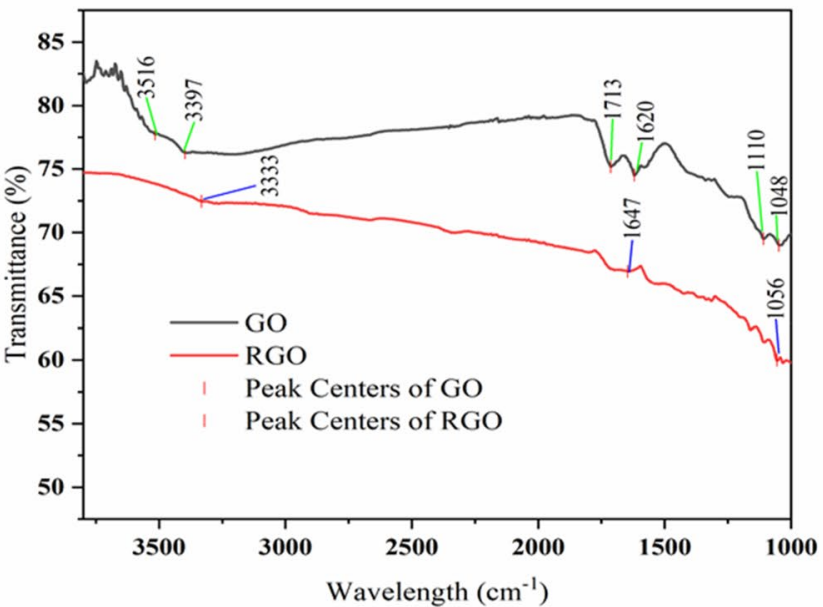

(b)

Fig. 2 a Raman spectra of synthesised GO and rGO samples (b) FTIR spectrum of synthesised GO and rGO 
rGO [32]. From the Fig. 2a, it was observed that the intensity ratios ( $\left.I_{D \text { band }} / I_{G \text { band }}\right)$ for $\mathrm{GO}$ were 0.6 while 0.64 for $\mathrm{rGO}$ which is slightly high than $\mathrm{GO}$, and showed the number of defects increases during reduction due to the restoration of numerous graphitic domains from the amorphous regions of graphite oxide as also reported in accordance with [31, 32].

The FTIR spectrum of synthesised GO is shown in Fig. $2 \mathrm{~b}$ and indicates the presence of $\mathrm{C}-\mathrm{O}$ (alkoxy) vibration stretch at $1048 \mathrm{~cm}^{-1}$ and $1110 \mathrm{~cm}^{-1} ; \mathrm{C}=\mathrm{C}$ vibration at $1620 \mathrm{~cm}^{-1} ; \mathrm{C}=\mathrm{O}$ vibration at $1713 \mathrm{~cm}^{-1}$ and $\mathrm{O}-\mathrm{H}$ stretch at $3397 \mathrm{~cm}^{-1}$. In the case of $\mathrm{rGO}$ sample the FTIR spectrum comprising minor peaks ay $1056 \mathrm{~cm}^{-1}, 3333 \mathrm{~cm}^{-1}$ while slightly strengthened peak at $1647 \mathrm{~cm}^{-1}$ as reported by Aboulkas et al. [33]. These observations confirmed the formation of $\mathrm{rGO}$.

The synthesised rGO was mixed in PEDOT: PSS solution using different concentrations $0 \%, 1 \%, 2 \%, 3 \%, 4 \%$, and $5 \%$ referred as S0, S1, S2, S3, S4, and S5 respectively. These solutions are sonicated for $4 \mathrm{~h}$ before deposition. The prepared solution was deposited on a PET sheet using a bar coating with a bar of wire size $30 \mu \mathrm{m}$. The sheet resistance of the deposited layer was measured. The alteration in sheet resistance with the $\mathrm{GO}$ concentration in PEDOT: PSS is reported in Fig. 3a. The sheet resistance at $\mathrm{rGO}$ concentration 1\% (wt\%) in PEDOT: PSS showed a sheet resistance of $315 \pm 8 \Omega /$ sq. which is the lowest among other samples.

The transmittance of all the samples S0, S1, S2, S3, S4, and $\mathrm{S} 5$ were measured using an absorption spectrophotometer as shown in Fig. 3b, and found that the optical transmittance of the $S 1$ in the visible region is higher in comparison to S2, S3, S4, and S5 samples. When the rGO concentration increases, the optical transmittance appears to be reduced. From Fig. 3, it is observed that the sample $\mathrm{S} 1$ has low sheet resistance ( $315 \pm 8 \Omega /$ sq.) and high transmittance $(\sim 74 \%)$ in comparison to the rest of the $r G O$ modified PEDOT: PSS samples shows a good combination for using as TCE. The combination of sheet resistance ( $315 \pm 8 \Omega /$ sq.) and transmittance $(\sim 74 \%)$ of $\mathrm{S} 1$ sample is much better than that was reported by Liu. et al. (sheet resistance $600 \Omega / \mathrm{sq}$. and transmittance $\sim 80 \%$ for Graphene/PEDOTP:SS) and Kiyoung et al. (sheet resistance $2.3 \mathrm{k} \Omega / \mathrm{sq}$. and transmittance $\sim 80 \%$ for rGO/PEDOT:PSS) in their individual work [34-36].

To analyse the uniformity or surface roughness of the deposited layer, AFM characterisation using Atomic Force Microscopy (AFM) using a machine of model Pro 47, provided by NT-MDT spectrum instruments Russia and optical images of the samples were recorded with an optical microscope (Olympus BX53M) as shown in Figs. 4, 5. The surface images reveal that the surface roughness increases with increasing the concentration of rGO in PEDOT: PSS. Sample $\mathrm{S} 1$ shows better quality in comparison to other samples including S2, S3, S4, and S5.

The flexibility and reliability of the deposited samples were tested by bending test using beakers of different diameters including 10, 8, 6, 4, 3 and $2 \mathrm{~cm}$ with bending curvature $20,25,33,50,67$, and $100 \mathrm{~m}^{-1}$. Both inner bending and outer bending of the samples were performed by the equation given below that defined the relative variation of electrical resistance [37]:

$\frac{\Delta R}{R_{0}}=\frac{\left(R-R_{0}\right)}{R_{0}}$

where $R_{0}$ is the resistance of the unbent sample [bending curvatures $(\rho)=0$ ] and R is the resistance of the bent sample at different bending curvatures $(\rho)$ values.

In this measurement, the variation in electrical resistance of the fabricated flexible transparent conducting

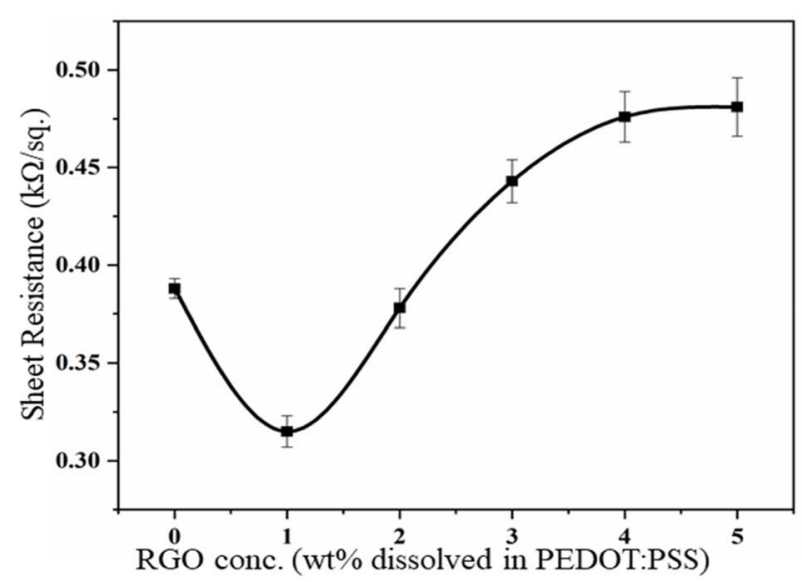

(a)

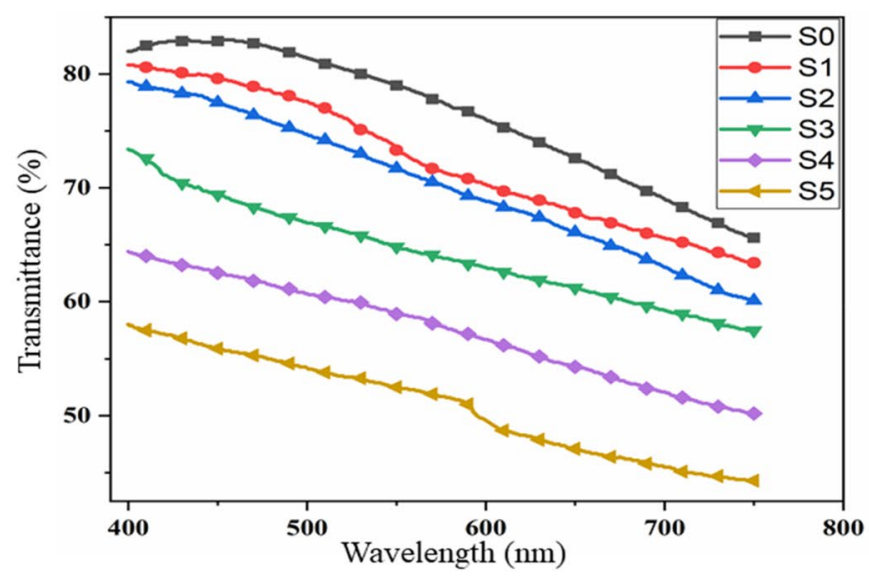

(b)

Fig. 3 a Sheet resistance of rGO modified PEDOT: PSS film $\mathbf{b}$ Transmittance of rGO modified PEDOT: PSS hybrid film 


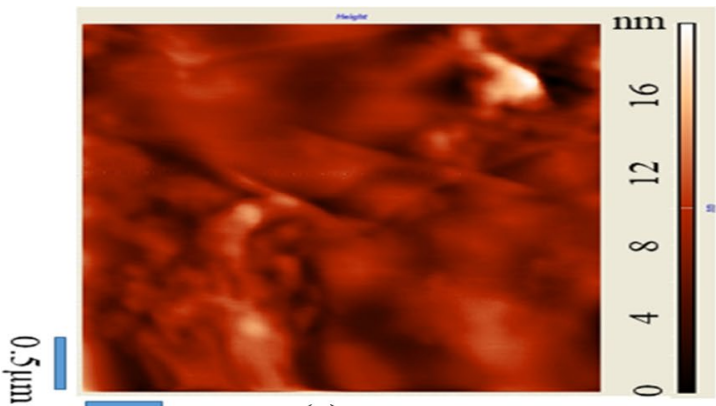

$$
0.5 \mu \mathrm{m}
$$

(a) $\mathrm{SO}$
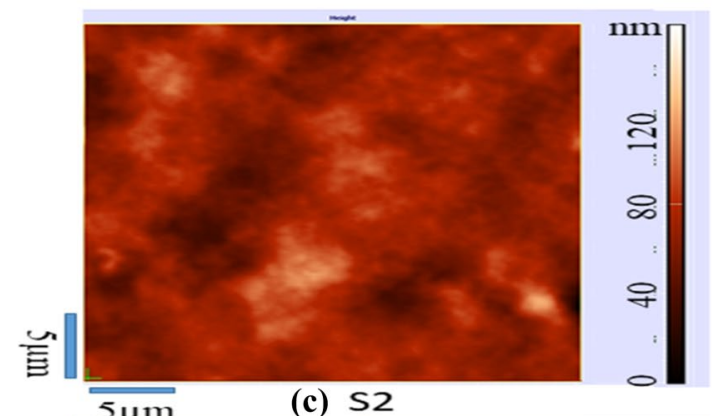

$$
\text { 㞫 }
$$

$5 \mu \mathrm{m}$

(c) 52

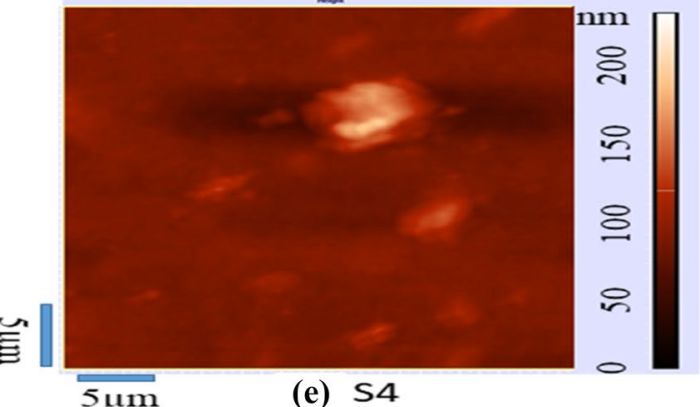

(e) 54

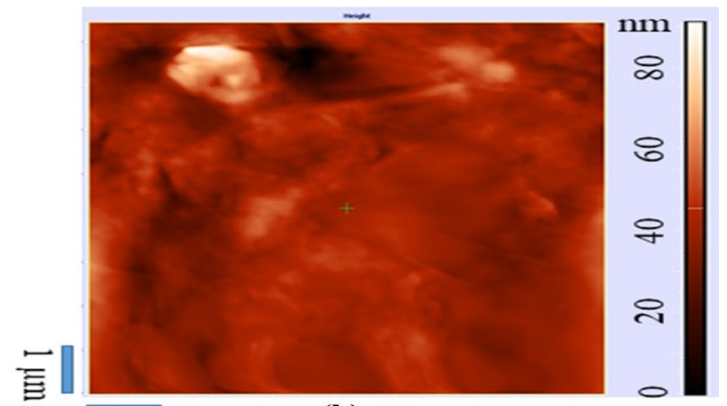

(b) $\mathrm{S1}$

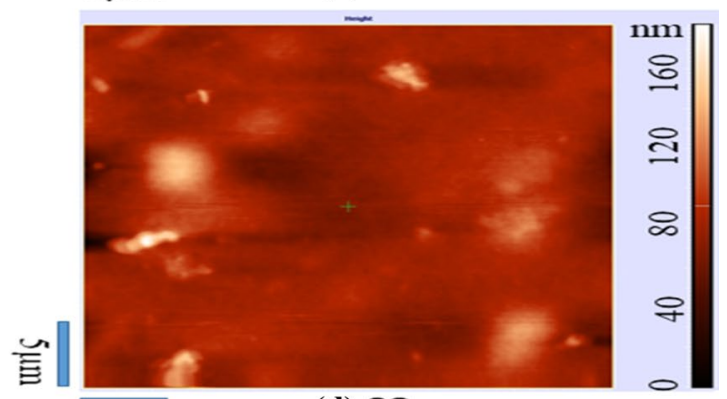

(d) $\mathrm{S3}$

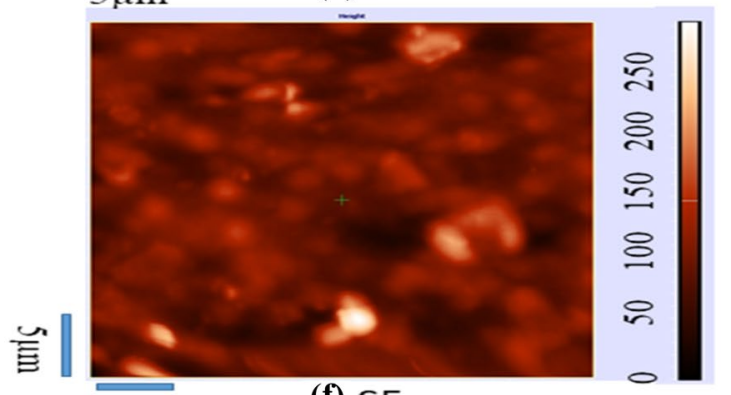

$5 \mu \mathrm{m}$

(f) $\mathrm{S5}$

Fig. 4 AFM images of the prepared samples

samples was measured and shown in Fig. 6. Sample S1 indicate the least variation in electrical resistance $(12 \%$ max. increases), in comparison to the other samples. Sample S2 indicate variation 15\% (increases); sample S3 showed variation 16.5\% (increases); sample S4 showed variation $\sim 17.4 \%$ (increases) and sample S5 showed variation $\sim 20 \%$ (increases) in electrical resistance for bending outer curvatures from 20 to $100 \mathrm{~m}^{-1}$ shown in Fig. 6a. The increase in resistance on outward bending is due to tensile stresses that tend to separate the layer particles from each other [38].

In a similar way, electrical resistance variation as a function of inward bending curvatures of rGO/PEDOT: PSS hybrid layer was measured and shown in Fig. 6b. Sample S1 shows minimum electrical resistance variation ( 9\% decreases), in comparison to the other prepared samples including sample S2 showed variation 12.5\%, sample S3 showed a variation 14\% (decreases), sample S4 showed a variation $\sim 16 \%$ (decreases) and sample S5 showed a variation $\sim 17 \%$ (decreases) in electrical resistance for inward bending curvatures from 20 to $100 \mathrm{~m}^{-1}$. The inward bending may create an overlap of the crumpled graphene films that results in a decrease in resistance [39].

Henceforth, the sample $\mathrm{S} 1$ is reported having the least sheet resistance $(315 \pm 8 \Omega /$ sq.), high transmittance $(\sim 74 \%)$, better smooth surface, and lowest variation in electrical resistance during the inward ( $9 \%$ max. decreases) and outward bending (12\% max. increases) in comparison to rest of the samples (S2, S3, S4, and S5). Hence, the sample S1 looked reliable and better for flexible TCE applications.

\section{Conclusions}

ITO is the most preferred TCE because of its high transmittance and low sheet resistance. However its brittle nature and limited indium resources restrict its use for future 


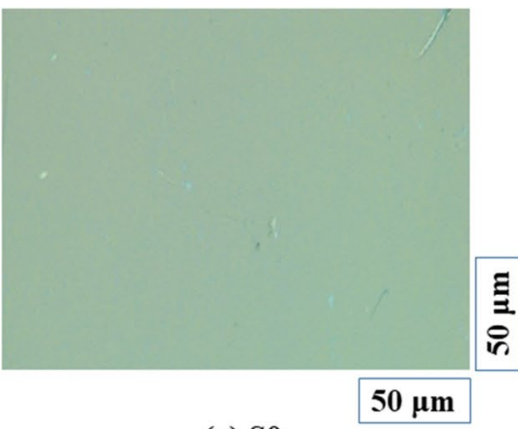

(a) S0

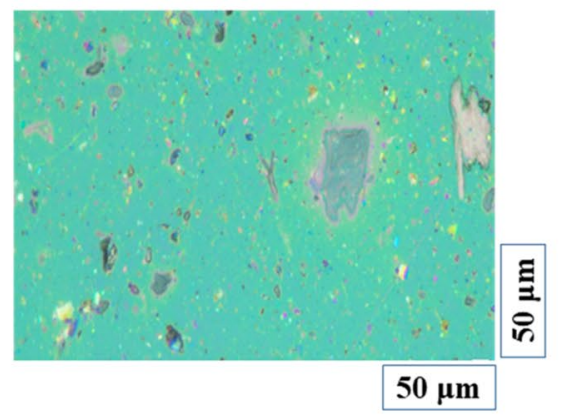

(d) $\mathrm{S3}$

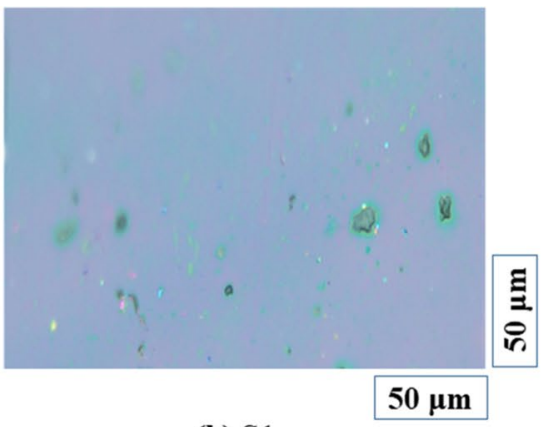

(b) S1

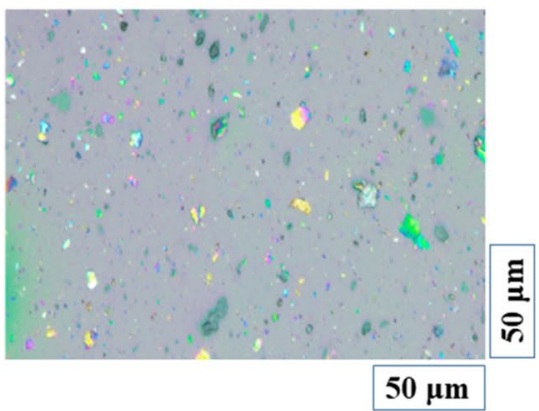

(e) $\mathrm{S} 4$

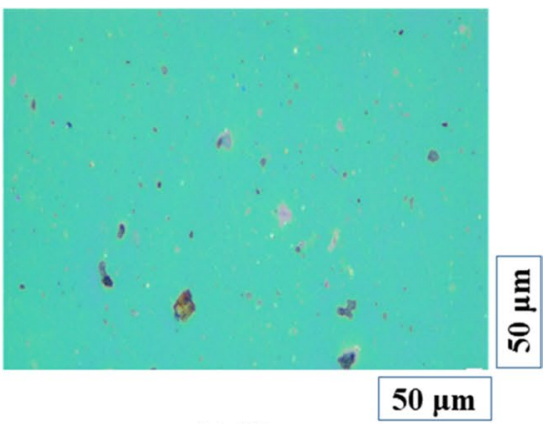

(c) S2

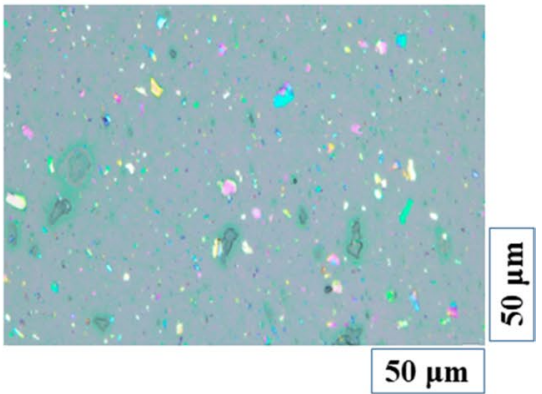

(f) $\mathrm{S5}$

Fig. 5 Optical microscopic images of the prepared samples

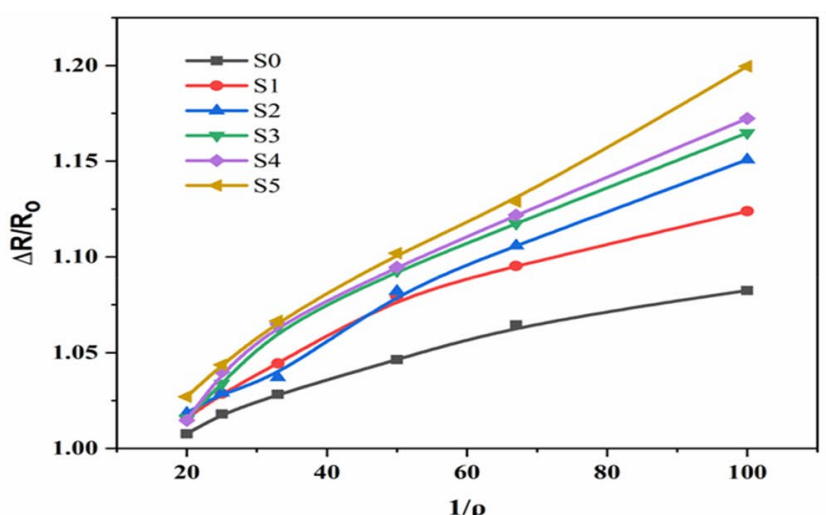

(a)

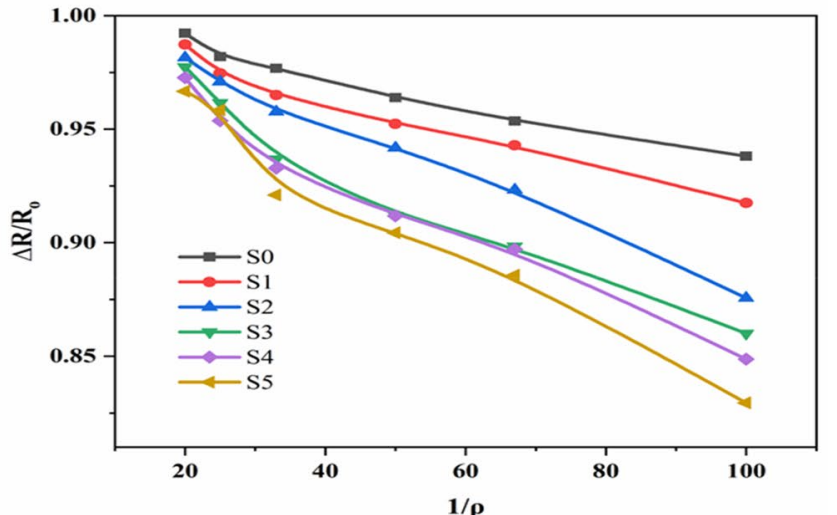

(b)

Fig. 6 a Variation in Electrical resistance of deposited samples for outer bending b Electrical resistance variation of deposited samples for inner bending

flexible optoelectronics devices. Hence, there is a requirement of alternate material for replacing ITO as TCE. Keeping this in view, the rGO modified PEDOT: PSS based TCE were deposited on a flexible PET sheet using a bar coating technique for achieving a flexible TCE as an alternate of ITO. The sheet resistance, optical transmittance, bending test (outside and inside bending) and surface roughness of the rGO modified PEDOT: PSS samples were measured. Our analysis reveals that the sample $S 1$, having a concentration of $\mathrm{rGO} 1 \mathrm{wt} \%$ in PEDOT: PSS, showing better results i.e. low sheet resistance $(315 \pm 8 \Omega /$ sq.) and high transmittance ( 74\%) with low electrical resistance variation $12 \%$ (max. increases) for outward bending and $9 \%$ (max. decreases) for inward bending of the sample for bending curvature from 20 to $100 \mathrm{~m}^{-1}$. Therefore, the sample with rGO $1 \mathrm{wt} \%$ in PEDOT: PSS is reported for its potential use as flexible TCE for optoelectronics applications.

Acknowledgements TEQIP-III, MHRD Government of India funded this work. 


\section{Compliance with ethical standards}

Conflicts of interest The authors declare that they have no conflict of interest.

Open Access This article is licensed under a Creative Commons Attribution 4.0 International License, which permits use, sharing, adaptation, distribution and reproduction in any medium or format, as long as you give appropriate credit to the original author(s) and the source, provide a link to the Creative Commons licence, and indicate if changes were made. The images or other third party material in this article are included in the article's Creative Commons licence, unless indicated otherwise in a credit line to the material. If material is not included in the article's Creative Commons licence and your intended use is not permitted by statutory regulation or exceeds the permitted use, you will need to obtain permission directly from the copyright holder. To view a copy of this licence, visit http://creativecommons .org/licenses/by/4.0/.

\section{References}

1. Song T-B, Li N (2014) Emerging transparent conducting electrodes for organic light emitting diodes. Electronics 3(1):190-204

2. Chu H-C et al (2016) Spray-deposited large-area copper nanowire transparent conductive electrodes and their uses for touch screen applications. ACS Appl Mater Interf 8(20):13009-13017

3. Lee $S$ et al (2020) Electrodeposited silver nanowire transparent conducting electrodes for thin film solar cells. ACS Appl Mater Interf 12:6169-6175

4. Sharma S, Shriwastava S, Kumar S, Bhatt K, Tripathi CC (2018) Alternative transparent conducting electrode materials for flexible optoelectronic devices. Opto-Electron Rev 26(3):223-235

5. López-Naranjo EJ, González-Ortiz LJ, Apátiga LM, Rivera-Muñoz EM, Manzano-Ramirez A (2016) Transparent electrodes: a review of the use of carbon-based nanomaterials. J Nanomater 2016:4928365. https://doi.org/10.1155/2016/4928365

6. Aronggaowa B, Kawasaki M, Shimomura T (2013) Thin, transparent conductive films fabricated from conducting polymer nanofibers. Polym J 45(8):819-823

7. Xia Y, Ouyang J (2015) Transparent conducting polymers. Organic Optoelectronic Materials. Springer, New York, pp 359-392

8. Gueye MN, Carella A, Faure-Vincent J, Demadrille R, Simonato J-P (2020) Progress in understanding structure and transport properties of PEDOT-based materials: a critical review. Prog Mater Sci 108:100616

9. Manawi Y, Samara A, Al-Ansari T, Atieh M et al (2018) A review of carbon nanomaterials' synthesis via the chemical vapor deposition (CVD) method". Materials (Basel) 11(5):822

10. Zaretski AV, Lipomi DJ (2015) Processes for non-destructive transfer of graphene: widening the bottleneck for industrial scale production. Nanoscale 7(22):9963-9969

11. Chandrashekar BN et al (2019) A universal stamping method of graphene transfer for conducting flexible and transparent polymers. Sci Rep 9(1):1-11

12. Chen $M$, Haddon RC, Yan R, Bekyarova E (2017) Advances in transferring chemical vapour deposition graphene: a review. Mater Horiz 4(6):1054-1063

13. Abad J, Padilla J (2018) Transparent conductive polymers . Trans Conduct Mater Mater Synth Charact Appl 29:193-244

14. Zhao P, Tang Q, Zhao X, Tong Y, Liu Y (2018) Highly stable and flexible transparent conductive polymer electrode patterns for largescale organic transistors. J Colloid Interf Sci 520:58-63

15. Wang Y et al (2017) A highly stretchable, transparent, and conductive polymer. Sci Adv 3(3):e1602076
16. Lombardo $\mathrm{V}$ et al (2018) Transparent conductive polymer obtained by in-solution doping of PEDOT: PSS. Polymer (Guildf) 155:199-207

17. Mengistie DA, Ibrahem MA, Wang P-C, Chu C-W (2014) Highly conductive PEDOT: PSS treated with formic acid for ITO-free polymer solar cells. ACS Appl Mater Interf 6(4):2292-2299

18. Mengistie DA, Wang P-C, Chu C-W (2013) Highly conductive PEDOT: PSS electrode treated with polyethylene glycol for ITOfree polymer solar cells. ECS Trans 58(11):49-56

19. Yildirim E et al (2018) A theoretical mechanistic study on electrical conductivity enhancement of DMSO treated PEDOT: PSS. J Mater Chem C 6(19):5122-5131

20. Rwei S-P, Lee Y-H, Shiu J-W, Sasikumar R, Shyr U-T (2019) Characterization of solvent-treated PEDOT: PSS thin films with enhanced conductivities. Polymers (Basel) 11(1):134

21. Nagata R, Yanagi Y, Fujii S, Kataura H, Nishioka Y (2014) Application of highly conductive DMSO-treated PEDOT: PSS electrodes to flexible organic solar cells, in 201421 st international workshop on active-matrix flatpanel displays and devices (AM-FPD) 299-302

22. Yoon S-S, Khang D-Y (2016) Roles of nonionic surfactant additives in PEDOT: PSS thin films. J Phys Chem C 120(51):29525-29532

23. Shi H, Liu C, Jiang Q, Xu J (2015) Effective approaches to improve the electrical conductivity of PEDOT: PSS: a review. Adv Electron Mater 1(4):1500017

24. Nagata R, Yanagi Y, Fujii S, Kataura H, Nishioka Y (2015) Highly conductive DMSO-treated PEDOT: PSS electrodes applied to flexible organic solar cells. IEICE Trans Electron 98(5):411-421

25. Liu G et al (2020) Solvent treatment inducing ultralong cycle stability poly (3, 4-ethylenedioxythiophene): poly (styrenesulfonic acid) fibers as binding-free electrodes for supercapacitors. Int J Energy Res 44(7):5856-5865

26. Liu J et al (2018) Highly conductive hydrogel polymer fibers toward promising wearable thermoelectric energy harvesting. ACS Appl Mater Interf 10(50):44033-44040

27. Alshammari AS (2019) Effect of solvents treatment on the electrical stability and surface wetting properties of PEDOT: PSS thin films. Mater Lett 250:30-33

28. Jørgensen M, Norrman K, Krebs FC (2008) Stability/degradation of polymer solar cells. Sol energy Mater Sol cells 92(7):686-714

29. Schultheiss A et al (2020) Insight into the degradation mechanisms of highly conductive poly (3, 4-ethylenedioxythiophene) thin films. ACS Appl Polym Mater 2:2686-2695

30. Lingstedt LV et al (2019) Effect of DMSO solvent treatments on the performance of PEDOT: PSS based organic electrochemical transistors. Adv Electron Mater 5(3):1800804

31. Pimenta MA, Dresselhaus G, Dresselhaus MS, Cancado LG, Jorio A, Saito R (2007) Studying disorder in graphite-based systems by Raman spectroscopy. Phys Chem Chem Phys 9(11):1276-1290

32. Patel DK, Hong P-D, Rathore S (2019) Surface-enhanced Raman scattering of reduced graphene coated with silver nanoparticles. J Ceram Process Res 20(4):442-448

33. Aboulkas A, Hammani H, El Achaby M, Bilal E, Barakat A et al (2017) Valorization of algal waste via pyrolysis in a fixed-bed reactor: production and characterization of bio-oil and bio-char. Bioresour Technol 243:400-408

34. Woo Y (2019) Transparent conductive electrodes based on graphene-related materials. Micromachines 10(1):13

35. Liu Z, Parvez K, Li R, Dong R, Feng X, Müllen K (2015) Transparent conductive electrodes from graphene/PEDOT: PSS hybrid inks for ultrathin organic photodetectors. Adv Mater 27(4):669-675

36. Jo K et al (2011) Stable aqueous dispersion of reduced graphene nanosheets via non-covalent functionalization with conducting polymers and application in transparent electrodes. Langmuir 27(5):2014-2018 
37. Sharma S et al (2020) Optical and electrical investigations of rubbing assisted few-layer graphene thin film for feasibility of flexible electrode. Optik (Stuttg) 203:163989

38. Luo S, Samad YA, Chan V, Liao K (2019) Cellular graphene: fabrication, mechanical properties, and strain-sensing applications. Matter 1(5):1148-1202

39. Chen $\mathrm{H}$ et al (2020) Enhanced stretchable and sensitive strain sensor via controlled strain distribution. Nanomaterials 10(2):218
Publisher's Note Springer Nature remains neutral with regard to jurisdictional claims in published maps and institutional affiliations.

\section{SN Applied Sciences}

a SPRINGER NATURE journal 